\title{
A dichotomy for P-ideals of countable sets
}

\author{
by
}

\author{
Stevo Todor čević (Paris)
}

\begin{abstract}
A dichotomy concerning ideals of countable subsets of some set is introduced and proved compatible with the Continuum Hypothesis. The dichotomy has influence not only on the Suslin Hypothesis or the structure of Hausdorff gaps in the quotient algebra $\mathcal{P}(\mathbb{N}) /$ fin but also on some higher order statements like for example the existence of Jensen square sequences.
\end{abstract}

The purpose of this note is to show that a dichotomy about P-ideals of countable subsets of $\omega_{1}$ appearing previously in a number of restricted contexts (see e.g., [1], [10], [11]) can be lifted without difficulties to the most general and optimal form:

(*) $\quad$ For every P-ideal $\mathcal{I}$ of countable subsets of some set $S$, either

(1) there is an uncountable $A \subseteq S$ such that $[A]^{\omega} \subseteq \mathcal{I}$, or

(2) $S$ can be decomposed into countably many sets orthogonal to $\mathcal{I}$.

Recall that an ideal $\mathcal{I}$ is a $P$-ideal if for every sequence $\left\{I_{n}\right\}$ of elements of $\mathcal{I}$ there is $J \in \mathcal{I}$ such that $I_{n} \backslash J$ is finite for all $n$. We shall consider here only ideals of countable subsets of some index set $S$ and we shall implicitly assume that every finite subset of $S$ belongs to the ideal we consider. A subset $B$ of $S$ is orthogonal to a family $\mathcal{F}$ of subsets of $S$ if $B \cap F$ is finite for every $F \in \mathcal{F}$. This notion of orthogonality comes from the classical writings of Hausdorff, Luzin and Rothberger about the gaps in the quotient algebra $\mathcal{P}(\mathbb{N}) /$ fin. This connection will be discussed in more detail below. The formulation of $(*)$, however, has its roots in a quite different area. This is the area that grew out of the famous problem of Suslin and studies the Ramsey-type properties of uncountable structures. We shall not reproduce here a historical analysis of this (which can be found e.g. in [1], [10], [11]) but instead concentrate on reproducing the analysis of $(*)$ and its consequences in the present unrestricted form.

2000 Mathematics Subject Classification: 03E05, 03E35, 03 E65. 
1. Suslin trees. This is an unrestricted version of $\S 2.1$ of [1]. A tree is a well-founded poset $\mathcal{T}=(T, \leq)$ which has the property that $\operatorname{pred}(t)=$ $\{s \in T: s \leq t\}$ is totally ordered for every $t \in T$. Identifying $\mathcal{T}$ with the family $P(T)=\{\operatorname{pred}(t): t \in T\}$ it is natural to let $(\mathcal{T})^{\perp}$ denote the ideal of all countable subsets of $T$ which are orthogonal to $P(T)$. Recall that a Cantor tree is any tree of height $\omega+1$ all of whose levels except the last one are countable.

Claim 1. $(\mathcal{T})^{\perp}$ is a P-ideal whenever $\mathcal{T}$ contains no Cantor subtrees.

Pr o of. Note that the equivalent formulation is that $(\mathcal{T})^{\perp}$ is a P-ideal whenever the family $P(T)$ is locally countable, i.e., it has a countable trace on any countable subset of $T$. So, given a sequence $\left\{I_{n}\right\}$ of elements of $(\mathcal{T})^{\perp}$ we let $I_{\omega}$ be the union of $I_{n}$ 's and let $J$ be a subset that interpolates the pregap formed by the two countable orthogonal families $\left\{I_{n}\right\}$ and the restriction of $P(T)$ to $I_{\omega}$.

Note that an uncountable subset $A$ of $T$ such that $[A]^{\omega} \subseteq(\mathcal{T})^{\perp}$ is a subtree of $\mathcal{T}$ of height $\leq \omega$, so the alternative $(1)$ for $(\mathcal{T})^{\perp}$ is equivalent to the fact that $T$ has an uncountable antichain. Note now that a subset of $T$ that is orthogonal to $(\mathcal{T})^{\perp}$ must be equal to the union of finitely many chains, so the alternative $(*)(2)$ is equivalent to the statement that $T$ can be decomposed into countably many chains. From this we conclude that $(*)$ implies the Suslin Hypothesis. However, one learns more from this example, the fact that in $(*)$ the hypothesis that $\mathcal{I}$ is $\mathrm{P}$-ideal is essential. To see this, let $\sigma \mathbb{Q}$ be the tree of all well-ordered subsets of the rationals ordered by end-extension and let $\mathcal{I}$ be the ideal of all countable subsets of $\sigma \mathbb{Q}$ that can be covered by finitely many sets from $P(\sigma \mathbb{Q})$. Note that $\mathcal{I}^{\perp} \cap[\sigma \mathbb{Q}]^{\omega}=(\sigma \mathbb{Q})^{\perp}$, so the dichotomy $(*)$ applied to $\mathcal{I}$ would say that either $\sigma \mathbb{Q}$ has an uncountable chain or it can be decomposed into countably many antichains. It is well known (see [8], §9) that neither of these two alternatives are true. It is known (see [10], [11]) that $(*)$ for non-P-ideals is true modulo restriction that the ideal be $\aleph_{1}$-generated. In this example this would mean that the tree would be of size $\aleph_{1}$ and then $(*)$ reduces to the well known statement that every tree of size $\aleph_{1}$ without uncountable chains is the union of countably many antichains (see [8], §9).

Forcing with a nontrivial measure algebra gives us a coherent sequence (see $\S 3$ below) which violates $(*)$. This has been shown in $\S 7$ of [11]. On the other hand, if $\mathcal{R}$ is a nontrivial measure algebra and if $\dot{\mathcal{T}}=\left(\theta, \leq_{\dot{T}}\right)$ is an $\mathcal{R}$-name for a tree on $\theta$ which contains no Cantor subtree then the family $\mathcal{I}$ of all countable $A \subseteq \theta$ such that

$$
\|\left\{\alpha \in A: \alpha \leq_{\dot{T}} \beta\right\} \text { is finite } \|=1
$$

for all $\beta \in \theta$ is a $\mathrm{P}$-ideal. It turns out that the $\mathrm{P}$-ideal $(\dot{\mathcal{T}})^{\perp}$ will not violate 
(*) in the forcing extension by $\mathcal{R}$ unless the P-ideal $\mathcal{I}$ already violates $(*)$ in the ground model. All this has been shown by J. Hirschorn in a recent article [4] during the course of proving that $(*)$ implies that the Suslin Hypothesis is preserved under forcing by any measure algebra. Hirschorn [4] also has a modification of $(*)$, with alternative (1) strengthened and alternative (2) weakened, which is tailored to imply the measure-algebra-forcing preservation of the statement that all Aronszajn trees are special, i.e., the union of countably many antichains:

$\left(*_{c}\right) \quad$ For every P-ideal $\mathcal{I}$ of countable subsets of some uncountable regular cardinal $\theta$, either

(1) there is a closed uncountable $C \subseteq \theta$ such that $[C]^{\omega} \subseteq \mathcal{I}$, or

(2) there is a stationary subset $S$ of $\theta$ which is orthogonal to $\mathcal{I}$.

It might be worth investigating which of the other consequences of $(*)$ that we list below allow randomization in this sense. In particular, it would be interesting to know whether the influence of $(*)$ on Hausdorff gaps, which we present in the next section, allows for randomization.

2. Hausdorff gaps. This is an unrestricted version of Section 2.2 of [1]. Let $\mathcal{A}$ and $\mathcal{B}$ be two orthogonal families of countable subsets of some set $S$, i.e., two families of sets with the property that every member of $\mathcal{A}$ has finite intersection with every member of $\mathcal{B}$. For the purpose of this example it is convenient to think of a countable subset $a$ of $S$ as given together with its enumeration, a bijection between an ordinal $\leq \omega$ and $a$. Thus, it is natural to use the notation $a[n]$ for the subset of $a$ consisting of the first $n$ members of $a$ in that fixed enumeration. With the pregap $(\mathcal{A}, \mathcal{B})$ we associate the ideal $\mathcal{I}=\mathcal{I}_{(\mathcal{A}, \mathcal{B})}$ of all countable subsets $B$ of $\mathcal{B}$ for which there exists $a \in \mathcal{A}$ such that

$$
B(a, n)=\{b \in B: b \cap a \subseteq a[n]\} \text { is finite for all } n<\omega .
$$

REmark. Note that if $x, y \in \mathcal{A}$ and $n \leq m$ are such that $(x \backslash y) \cup$ $(y[n] \cap x) \subseteq x[m]$ then $B(y, n)$ is included in $B(x, m)$. So, if $x$ is almost included in $y$, then for every $n$ there is $m$ such that $B(y, n)$ is included in $B(x, m)$. From this we conclude that if an $a \in \mathcal{A}$ witnesses the condition (i) for a given $B \in \mathcal{I}$ then so does any $a^{\prime} \in \mathcal{A}$ which almost includes $a$. A similar argument shows that the definition of $\mathcal{I}$ does not depend on the choice of enumerations of countable subsets of $S$.

Claim 2. $\mathcal{I}_{(\mathcal{A}, \mathcal{B})}$ is a P-ideal whenever $\mathcal{A}$ is $\sigma$-directed under almost inclusion.

P r o of. Let $\left\{B_{k}\right\}$ be a given sequence of elements of $\mathcal{I}$ and let $\left\{a_{k}\right\}$ be the corresponding sequences from $\mathcal{A}$. Since $\mathcal{A}$ is $\sigma$-directed, pick $a \in \mathcal{A}$ such 
that $a_{k} \backslash a$ is finite for all $k$. From the Remark we know that $B_{k}(a, n)$ is finite for all $n, k<\omega$. For $n<\omega$, set

$$
B_{n}^{\prime}=B_{n} \backslash B_{n}(a, n) \text {. }
$$

Let $B=\bigcup_{n<\omega} B_{n}^{\prime}$. Then $B$ almost includes each $B_{n}$ and $a$ witnesses its membership in $\mathcal{I}_{(\mathcal{A}, \mathcal{B})}$.

Claim 3. If $X \subseteq \mathcal{B}$ is orthogonal to $\mathcal{I}_{(\mathcal{A}, \mathcal{B})}$ then $c=\bigcup X$ is orthogonal to $\mathcal{A}$.

Pro of. Suppose $a \cap c$ is infinite for some $a \in \mathcal{A}$. So for each $n<\omega$ we can fix $b_{n} \in X$ such that $a \cap b_{n} \nsubseteq a[n]$. It follows that $\left\{b_{n}: n<\omega\right\}$ is an infinite subset of $X$ that belongs to $\mathcal{I}$, a contradiction.

Recall that two families $a_{\xi}\left(\xi<\omega_{1}\right)$ and $b_{\xi}\left(\xi<\omega_{1}\right)$ of countable sets indexed by $\omega_{1}$ form a Hausdorff gap if they are orthogonal to each other and if they satisfy the following condition that shows up in Hausdorff's original construction of an $\left(\omega_{1}, \omega_{1}^{*}\right)$-gap in the quotient algebra $\mathcal{P}(\mathbb{N}) /$ fin (see [3]):

$$
\left\{\xi<\alpha: a_{\alpha} \cap b_{\xi} \subseteq a_{\alpha}[n]\right\} \text { is finite for all } \alpha<\omega_{1} \text { and } n<\omega .
$$

Clearly, if $a_{\xi}\left(\xi<\omega_{1}\right)$ and $b_{\xi}\left(\xi<\omega_{1}\right)$ form a Hausdorff gap then they are inseparable in a rather strong sense: For every subset $X$ of $\bigcup_{\xi<\omega_{1}}\left(a_{\xi} \cup b_{\xi}\right)$ one of the sets

$$
\left\{\xi<\omega_{1}: a_{\xi} \perp X\right\} \quad \text { or } \quad\left\{\xi<\omega_{1}: b_{\xi} \subseteq X\right\}
$$

must be countable.

ClaIm 4. If there is an uncountable $X \subseteq \mathcal{B}$ such that $[X]^{\omega} \subseteq \mathcal{I}_{(\mathcal{A}, \mathcal{B})}$ then $(\mathcal{A}, \mathcal{B})$ contains a Hausdorff subgap.

Proof. Let $b_{\xi}\left(\xi<\omega_{1}\right)$ be a one-to-one sequence of elements of $X$. For each $\alpha<\omega$, pick $a_{\alpha} \in \mathcal{A}$ witnessing the fact that $\left\{b_{\xi}: \xi<\alpha\right\}$ belongs to $\mathcal{I}_{(\mathcal{A}, \mathcal{B})}$

Applying $(*)$ to the ideal $\mathcal{I}_{(\mathcal{A}, \mathcal{B})}$ we get the following consequence reminiscent of the influence of the Open Coloring Axiom on the gaps in $\mathcal{P}(\mathbb{N}) /$ fin (see [10], §8):

$\left(*_{g}\right) \quad$ If $\mathcal{A}$ and $\mathcal{B}$ are two orthogonal families of countable subsets of some set $S$ and if $\mathcal{A}$ is $\sigma$-directed under almost inclusion, then either

(1) $(\mathcal{A}, \mathcal{B})$ contains a Hausdorff subgap, or

(2) there is a countable family $\left\{S_{n}\right\}$ of subsets of $S$ which are orthogonal to $\mathcal{A}$ such that every element of $\mathcal{B}$ is included in one of the $S_{n}$ 's. 
Note that $\left(*_{g}\right)$ has an element of reflection which says that if $(\mathcal{A}, \mathcal{B})$ cannot be separated as in (2) then it contains a subgap of size $\aleph_{1}$ which also cannot be separated as in (2). We have already remarked that this reflection statement for $\mathcal{A}, \mathcal{B} \subseteq \mathcal{P}(\mathbb{N})$ is true under OCA with no restriction that $\mathcal{A}$ must be a P-ideal but of course changing the alternative (2) to the statement that there is a sequence $\left\{S_{n}\right\}$ of subsets of $S$ such that for every $a \in \mathcal{A}$ and $b \in \mathcal{B}$ there exists $n$ such that $a \perp S_{n}$ and $b \subseteq S_{n}$ (a statement which is equivalent to (2) when $\mathcal{A}$ is $\sigma$-directed under almost inclusion). Unfortunately, this unrestricted reflection, the main source of strength of OCA, cannot be stretched to the context of an arbitrary set $S$ in place of $\mathbb{N}$ at least if we are looking for a statement compatible with $\mathrm{GCH}$ or standard Forcing Axioms. For example, many of these axioms imply the existence of a regular uncountable cardinal $\theta$ which carries a diamond sequence concentrating on cofinality omega ordinals. Using the diamond sequence one easily constructs two orthogonal families $\mathcal{A}$ and $\mathcal{B}$ of subsets of $\theta$ of order type $\omega$ such that

(a) $F \mapsto \sup (F)$ is one-to-one on $\mathcal{A} \cup \mathcal{B}$,

(b) every unbounded subset of $\theta$ contains a member of $\mathcal{A}$ as well as a member of $\mathcal{B}$.

Thus, $\mathcal{A}$ and $\mathcal{B}$ cannot be separated even though, using a bit of Martin's axiom, any two subfamilies of $\mathcal{A}$ and $\mathcal{B}$ of size $\aleph_{1}$ can be separated. Note also that $\mathcal{I}=(\mathcal{A})^{\perp} \cap[\theta]^{\omega}$ is a P-ideal of countable subsets of $\theta$ which shows that in $(*)$ we cannot switch the alternatives $(1)$ and (2), at least if we would like to be compatible with, say, GCH.

3. Coherent sequences. This is an unrestricted version of $\S 2.3$ of [1]. Hausdorff's original $\left(\omega_{1}, \omega_{1}^{*}\right)$-gap can be considered as a sequence $f_{\alpha}: A_{\alpha} \rightarrow 2$ $\left(\alpha<\omega_{1}\right)$ of partial functions on $\mathbb{N}$ whose domains $A_{\alpha}\left(\alpha<\omega_{1}\right)$ form an increasing sequence relative to the inclusion $\subseteq^{*}$ modulo a finite set and which is coherent in the sense that $f_{\alpha}={ }^{*} f_{\beta}\left\lceil A_{\alpha}\right.$. (Here $={ }^{*}$ denotes equality modulo a finite set.) A family of coherent partial functions may be coherent for the trivial reason that there is a global function which induces them all modulo finite changes. On the other hand such a global function cannot be found if the family contains an uncountable subfamily of pairwise incompatible functions. The coherent sequence $f_{\alpha}: A_{\alpha} \rightarrow 2$ of Hausdorff [3] is nontrivial exactly for this reason since his condition (ii) presented above in $\S 2$ implies that every uncountable subsequence of $\left\{f_{\alpha}\right\}$ can be refined to an uncountable sequence of pairwise incompatible functions. The dichotomy which says that one of these two alternatives happens for every family of partial functions from $\mathbb{N}$ into 2 is one of the reformulations of the Open Coloring Axiom. It is therefore natural to search for a similar dichotomy about partial functions on an arbitrary set rather than $\mathbb{N}$. Again some restriction 
on the family needs to be made. One of the natural restrictions is that the coherent family $\mathcal{F}$ of partial functions can be written as

$$
\mathcal{F}=\left\{f_{A}: A \rightarrow 2: A \in \mathcal{A}\right\}
$$

where $\mathcal{A}$ is a family of countable subsets of some set $S$ which is $\sigma$-directed under the relation $\subseteq^{*}$. From the coherence we see that

$$
\mathcal{A}_{0}=\left\{f_{A}^{-1}(0): A \in \mathcal{A}\right\} \quad \text { and } \quad \mathcal{A}_{1}=\left\{f_{A}^{-1}(1): A \in \mathcal{A}\right\}
$$

are orthogonal to each other. The assumption that $\mathcal{A}$ is $\sigma$-directed gives us that both $\mathcal{A}_{0}$ and $\mathcal{A}_{1}$ are $\sigma$-directed. Note that the alternative $\left(*_{g}\right)(2)$ gives us a total function $g: S \rightarrow 2$ such that $g\left\lceil A={ }^{*} f_{A}\right.$ for all $A \in \mathcal{A}$. The alternative $\left(*_{g}\right)(1)$, on the other hand, gives us an uncountable subfamily of $\mathcal{F}$ of pairwise incompatible functions. In other words, we have the following:

$\left(*_{h}\right) \quad$ A coherent family $\mathcal{F}=\left\{f_{A}: A \rightarrow 2: A \in \mathcal{A}\right\}$ of partial functions indexed by some $\sigma$-directed family $\mathcal{A}$ of countable subsets of some set $S$ is induced by a single total function $g: S \rightarrow 2$ if and only if it contains no uncountable subfamily of pairwise incompatible functions.

The full power of $(*)$ itself is being used, however, when one considers some weaker forms of coherence of partial functions. We shall say that two partial functions $f_{A}: A \rightarrow \omega$ and $f_{B}: B \rightarrow \omega$ are weakly coherent if for every $X \subseteq A \cap B$, the function $f_{A}$ is unbounded on $X$ iff the function $f_{B}$ is unbounded on $X$.

$\left(*_{d}\right) \quad$ For every family $\mathcal{F}=\left\{f_{A}: A \rightarrow \omega: A \in \mathcal{A}\right\}$ of weakly coherent functions indexed by some $\sigma$-directed family $\mathcal{A}$ of countable subsets of some set $S$, either

(1) there is an uncountable $X \subseteq S$ such that $f\lceil X$ is finite-to-one for every $f \in \mathcal{F}$, or

(2) $S$ can be decomposed into countably many sets on which each of the functions from $\mathcal{F}$ is bounded.

To see that $\left(*_{d}\right)$ follows from $(*)$ let $\mathcal{I}$ be the family of all countable subsets $I$ of $S$ for which there exists $A \in \mathcal{A}$ which almost includes $I$ such that the function $f_{A}$ is finite-to-one on $I$.

Claim 5. $\mathcal{I}$ is a P-ideal.

P r o o f. Given a sequence $\left\{I_{n}\right\}$ of elements of $\mathcal{I}$ let $\left\{A_{n}\right\}$ be the sequence of witnesses from $\mathcal{A}$. Since $\mathcal{A}$ is $\sigma$-directed, pick $A \in \mathcal{A}$ such that $A_{n} \backslash A$ is finite for all $n$. For $n<\omega$, set

$$
I_{n}^{\prime}=\left\{x \in I_{n} \cap A: f_{A}(x) \geq n\right\} .
$$


Note that $I_{n} \backslash I_{n}^{\prime}$ is a finite set by the weak coherence of the family $\mathcal{F}$. It is clear that $I=\bigcup_{n<\omega} I_{n}^{\prime}$ is a member of $\mathcal{I}$ as witnessed by $A \in \mathcal{A}$.

Applying $(*)$ to $\mathcal{I}$ we get immediately the alternatives (1) and $(2)$ of $\left(*_{d}\right)$ from the corresponding alternatives of $(*)$.

4. Square sequences. For an ordinal $\theta$ (typically regular and uncountable) a $C$-sequence on $\theta$ is simply a sequence of the form $C_{\alpha}(\alpha<\theta)$ such that $C_{\alpha+1}=\{\alpha\}$ and $C_{\alpha}$ is a closed unbounded subset of $\alpha$ when $\alpha$ is limit. The coherence in this context means that $C_{\alpha}=C_{\beta} \cap \alpha$ whenever $\alpha$ is a limit point of $C_{\beta}$. When a given $C$-sequence has this coherence property then it is usually called Jensen's square sequence (see [5]), in which case one usually assumes that the sequence satisfies some sort of nontriviality condition such as, for example, the nonexistence of a closed unbounded subset $C$ of $\theta$ such that $C_{\alpha}=C \cap \alpha$ whenever $\alpha$ is a limit point of $C$. With a given $C$-sequence $C_{\alpha}(\alpha<\theta)$ one usually associates the notion of a walk from an ordinal $\beta$ to some smaller ordinal $\alpha$ along this sequence and defines various distance functions (see [9]). Here, we shall be interested in the simplest one, the function that counts the number of steps in the walk. Formally, define $\varrho_{2}:[\theta]^{2} \rightarrow \omega$ recursively by

$$
\varrho_{2}(\alpha, \beta)=\varrho_{2}\left(\alpha, \min \left(C_{\beta} \backslash \alpha\right)\right)+1 .
$$

The nontriviality of the square sequence $C_{\alpha}(\alpha<\theta)$ in case $\theta$ is a regular uncountable cardinal can be reformulated as follows (see [9], 1.14(c)).

(iii) For every family $\mathcal{F}$ of size $\theta$ whose members are pairwise disjoint finite subsets of $\theta$ and for every integer $n$ there exist $a$ and $b$ in $\mathcal{F}$ such that $\varrho_{2}(\alpha, \beta)>n$ whenever $\alpha \in a$ and $\beta \in b$.

The coherence condition on the square sequence $C_{\alpha}(\alpha<\theta)$ gives us the following notion of coherence between the functions $\left(\varrho_{2}\right)_{\alpha}=\varrho_{2}(\cdot, \alpha): \alpha \rightarrow \omega$ (see [9], 1.14(a)):

$$
\sup _{\xi<\alpha}\left|\varrho_{2}(\xi, \alpha)-\varrho_{2}(\xi, \beta)\right|<\infty \quad \text { for every } \alpha<\beta<\theta .
$$

The influence of $(*)$ on Jensen's notion of square sequences can now be stated as follows:

$\left(*_{e}\right) \quad$ Every coherent $C$-sequence defined on some regular uncountable cardinal other than $\omega_{1}$ must be trivial.

To see this, consider the ideal $\mathcal{I}$ of all countable subsets $I$ of $\theta$ such that each of the mappings $\left(\varrho_{2}\right)_{\alpha}$ is finite-to-one on $I$. Note that (iv) in particular means that $\left(\varrho_{2}\right)_{\alpha}(\alpha<\theta)$ is a weakly coherent family of functions, so as above we conclude that $\mathcal{I}$ is a P-ideal of countable subsets of $\theta$. The 
alternative $(*)(1)$ for this $\mathcal{I}$ is clearly impossible by our assumption about the cofinality of $\theta$. The alternative $(*)(2)$, on the other hand, gives us an unbounded subset $X$ of $\theta$ on which each of the functions $\left(\varrho_{2}\right)_{\alpha}$ is bounded and this in particular means that (iii) fails. From this we conclude that the given $C$-sequence $C_{\alpha}(\alpha<\theta)$ must be trivial, i.e., there must be a closed unbounded subset $C$ of $\theta$ such that $C \cap \alpha=C_{\alpha}$ for all limit points $\alpha$ of $C$.

This can be seen more directly as follows. Given the set $X$ we first find an unbounded subset $Y$ of $\theta$ and an integer $n$ such that $\varrho_{2}(\alpha, \beta) \leq n$ whenever $\alpha \in X, \beta \in Y$ and $\alpha<\beta$. Suppose that $n$ is the minimal integer for which one can find two such sets $X$ and $Y$. If the $C$-sequence is nontrivial then for each large enough limit ordinal $\xi$ in $\theta$ there must be $\beta(\xi)$ in $Y$ above $\xi$ such that $\xi$ is not a limit point of $C_{\beta(\xi)}$. It follows that there is an ordinal $f(\xi)<\xi$ such that the walk from $\beta(\xi)$ to any ordinal $\alpha$ in the interval $(f(\xi), \xi)$ has the walk from $\xi$ to $\alpha$ as a proper tail. Applying the Pressing Down Lemma to the regressive map $f$ gives us a stationary set $S$ of limit ordinals of $\theta$ such that $f$ takes some constant value $\eta$ on $S$. Let $X_{0}$ be the tail of $X$ above $\eta$ and let $Y_{0}=S$. Then $X_{0}$ and $Y_{0}$ are unbounded subsets of $\theta$ on whose product the function $\varrho_{2}$ is bounded by $n-1$, contradicting the minimality of $n$.

The proof that the statement (iii) is equivalent to the nontriviality of the $C$-sequence is very similar and so the reader is urged to supply the proof of this equivalence using the same basic idea.

Note that on $\omega_{1}$ there is always a nontrivial coherent $C$-sequence. It suffices to take for every limit ordinal $\alpha<\omega_{1}$ a subset $C_{\alpha}$ of order type $\omega$ which is cofinal in $\alpha$. The coherence condition is vacuously true as no $C_{\alpha}$ has limit points. From $\left(*_{e}\right)$ we conclude that the dichotomy $(*)$ implies that Jensen's square principle fails on any regular cardinal $>\omega_{1}$. By recent results from the Inner Model Theory we learn that any consistency proof of $(*)$ must involve considerably strong large cardinal assumptions. In the next section we shall see that indeed the consistency of the existence of a supercompact cardinal gives us the consistency of $(*)$.

The above proof suggests the following preordering between partial integer-valued functions: $f \leq^{0} g$ iff $\operatorname{dom}(f) \subseteq \operatorname{dom}(g)$ and $g$ is unbounded on any subset of $\operatorname{dom}(f)$ on which $f$ is unbounded. Note that the condition (iv) in particular means that $\left(\varrho_{2}\right)_{\alpha}(\alpha<\theta)$ is an $\leq^{0}$-increasing sequence of functions. The condition (iii) on the other hand implies that the sequence has no upper bound. There is another more elementary example of an $\leq^{0}$-increasing and $\leq^{0}$-unbounded sequence. Namely consider any sequence $e_{\alpha}: \alpha \rightarrow \omega\left(\alpha<\omega_{1}\right)$ of finite-to-one mappings. However, since $\omega_{1}$ is the maximal length of any sequence of finite-to-one mappings it is natural to ask for a longer sequence that would be $\leq^{0}$-increasing and $\leq^{0}$-unbounded for more subtle reasons. The following dichotomy about increasing sequences 
in this ordering shows that the prototype example of finite-to-one mappings must, in fact, be present in any case when there is unboundedness:

$\left(*_{f}\right) \quad$ For every $\leq^{0}$-increasing sequence $f_{\alpha}: \alpha \rightarrow \omega(\alpha<\theta)$, either

(1) there is an uncountable $X \subseteq \theta$ such that $f_{\alpha}\lceil X$ is finite-to-one for all $\alpha<\theta$, or

(2) there is $g: \theta \rightarrow \omega$ such that $f_{\alpha} \leq^{0} g$ for all $\alpha<\theta$.

To see that $\left(*_{f}\right)$ follows from $(*)$, assume $\theta$ has uncountable cofinality (or else $\left(*_{f}\right)(2)$ is easily satisfied) and consider the ideal $\mathcal{I}$ of all countable $I \subseteq \theta$ such that $f_{\alpha}\lceil I$ is finite-to-one for all sufficiently large $\alpha$. Using the assumptions that $\theta$ has uncountable cofinality and that the sequence of $f_{\alpha}$ 's is $\leq{ }^{0}$-increasing, it is not hard to show that the ideal $\mathcal{I}$ is a P-ideal. Now we apply $(*)$ to $\mathcal{I}$. It is clear that the alternative $(*)(1)$ gives us the set $X \subset \theta$ satisfying $\left(*_{f}\right)(1)$, which in turn implies that $\theta$ has cofinality exactly $\omega_{1}$. On the other hand, if $\theta=\bigcup_{n=0}^{\infty} S_{n}$ is a disjoint decomposition of $\theta$ into sets that are orthogonal to $\mathcal{I}$, the function $g: \theta \rightarrow \omega$ that is constantly equal to $n$ on $S_{n}$ would bound all $f_{\alpha}$ 's.

5. Consistency. The purpose of this section is to show both that $(*)$ is a consequence of the Proper Forcing Axiom as well as that it is consistent with GCH relative to the consistency of the supercompact cardinal. The global structure of this consistency proof follows the outline of Baumgartner's proof of the consistency of PFA via Laver's supercompactness sequence (see [2], [6]) except that instead of using Shelah's Proper-Forcing Iteration Lemma we use results of Shelah $[7, \mathrm{~V}]$ that forcing notions with certain completeness properties can be iterated without adding reals. Thus, the actual proof of consistency of $(*)$ with $(\mathrm{G}) \mathrm{CH}$ is done using a countable-support iteration up to a supercompact cardinal where at a successor stage we force with a proper and complete forcing notion that takes care of a particular instance of $(*)$.

Given a forcing notion $\mathcal{P}$ and a countable elementary submodel $M$ of some large enough structure of the form $H_{\kappa}$, we say that a condition $q$ of $\mathcal{P}$ is $M$-generic if it forces that the generic filter intersects $\mathcal{D} \cap M$ for every dense open subset $\mathcal{D}$ of $\mathcal{P}$ which belongs to $M$. The properness of the forcing notion $\mathcal{P}$ is the requirement that such a condition $q$ can always be found. Note however the stronger requirement that one can put on $q$ : that $q$ should extend every condition of some filter of $\mathcal{P} \cap M$ intersecting every dense open subset of $\mathcal{P}$ belonging to the submodel $M$. Having such a condition $q$ for every sufficiently large countable elementary submodel ensures not only that $\mathcal{P}$ is proper but also that it introduces no new reals. This in fact will be our goal when designing our basic forcing notion that deals with a particular instance of $(*)$. The forcing notion that we are just about to describe is a 
natural generalization of one from [1] designed for proving the consistency of $(*)$ on $\omega_{1}$.

Our input is a nonprincipal $\mathrm{P}$-ideal $\mathcal{I}$ on some ordinal $\theta$ which has the property that $\theta$ cannot be decomposed into countably many sets orthogonal to $\mathcal{I}$ but every smaller ordinal can. Note that this in particular implies that $\theta$ has uncountable cofinality. The poset $\mathcal{P}=\mathcal{P}_{\mathcal{I}}$ is a natural poset designed to force an uncountable subset $A$ of $\theta$ such that $[A]^{\omega} \subseteq \mathcal{I}$. Thus, the conditions of $\mathcal{P}$ are pairs $p=\left\langle x_{p}, \mathfrak{X}_{p}\right\rangle$ where:

(v) $\quad x_{p}$ is a countable subset of $\theta$,

(vi) $\mathfrak{X}_{p}$ is a countable collection of cofinal subsets of $[\mathcal{I}]^{\omega}$ when considered as a partial ordering ordered by the relation $\subseteq$.

Before defining the ordering of $\mathcal{P}$ let $<_{w}$ be a fixed well-ordering of $\mathcal{I}$. For $a \in[\mathcal{I}]^{\omega}$ let $A_{a}$ be the $<_{w}$-minimal member $C$ of $\mathcal{I}$ such that $B \subseteq^{*} C$ for all $B$ in $a$ and such that $C$ is covered by the union of the $B$ 's from $a$. The ordering on $\mathcal{P}$ is defined by letting $q \leq p$ ( $q$ extends $p$ ) when

(vii) $x_{q}$ end-extends $x_{p}$,

(viii) $\mathfrak{X}_{p} \subseteq \mathfrak{X}_{q}$,

(ix) for every $X \in \mathfrak{X}_{p}$ the set $\left\{a \in X: x_{q} \backslash x_{p} \subseteq A_{a}\right\}$ is cofinal in $[\mathcal{I}]^{\omega}$ and it belongs to $\mathfrak{X}_{q}$.

Lemma 1. For every $p \in \mathcal{P}$ and $\gamma<\theta$ there is an extension $q$ of $p$ such that $x_{q} \backslash \gamma \neq \emptyset$.

Proof. Otherwise, for every $\beta \in \theta \backslash \gamma$ there is $X \in \mathfrak{X}_{p}$ such that

$$
X(\beta)=\left\{a \in X: \beta \in A_{a}\right\}
$$

is not cofinal in $[\mathcal{I}]^{\omega}$. For a given $X \in \mathfrak{X}_{p}$, let $B(X)$ be the set of all $\beta \in \theta \backslash \gamma$ for which $X(\beta)$ is not cofinal in $[\mathcal{I}]^{\omega}$. Our assumption is that the family $B(X)\left(X \in \mathfrak{X}_{p}\right)$ covers $\theta \backslash \gamma$ so we shall reach a contradiction once we show that each $B(X)$ is orthogonal to $\mathcal{I}$. For suppose that for some $X \in \mathfrak{X}_{p}$ there is a countably infinite set $B \subseteq B(X)$ belonging to $\mathcal{I}$. Since $\mathcal{I}$ is a P-ideal and $X$ is cofinal in $[\mathcal{I}]^{\omega}$, removing a finite set from $B$, we may assume that

$$
Y=\left\{a \in X: B \subseteq A_{a}\right\}
$$

is cofinal in $[\mathcal{I}]^{\omega}$. Note that $Y \subseteq X(\beta)$ for each $\beta \in B$, so $Y$ is not supposed to be cofinal in $\mathcal{I}$ because no $X(\beta)$ is, a contradiction.

Lemma 2. $\mathcal{P}$ is proper.

P r o of. We are given a countable elementary submodel $M$ of some large enough structure $H_{\kappa}$ such that $\theta,<_{w}, \mathcal{I}, \mathcal{P} \in M$. We are also given $p_{0} \in$ $\mathcal{P} \cap M$. Let $\left\{\mathcal{D}_{n}\right\}$ be an enumeration of all dense open subsets of $\mathcal{P}$ that are members of $M$. Starting from $p_{0}$ we build a chain of conditions $p_{n}=\left(x_{n}, \mathfrak{X}_{n}\right)$ $(n<\omega)$ such that $p_{n+1} \in \mathcal{D}_{n} \cap M$ for all $n$. We shall find a condition $q$ 
extending all $p_{n}$ 's. Having in mind a stronger completeness property of $\mathcal{P}$, we consider an arbitrary set $B \subseteq M \cap \theta$ which has the property that $A \subseteq \subseteq^{*} B$ for all $A \in \mathcal{I} \cap M$. We shall try to construct the sequence $\left\{p_{n}\right\}$ in such a way that the following two conditions are satisfied when we let $x_{\omega}=\bigcup_{n<\omega} x_{n}$ :

(x) $\quad x_{\omega} \backslash x_{0} \subseteq B$,

(xi) for every $n<\omega$ and $X \in \mathfrak{X}_{n}$, the set $Z(X, n)=\left\{a \in X: x_{\omega} \backslash x_{n} \subseteq A_{a}\right\}$ is cofinal in $[\mathcal{I}]^{\omega}$.

If this can be done then $q=\left(x_{q}, \mathfrak{X}_{q}\right)$ defined by

$$
x_{q}=x_{\omega}, \quad \mathfrak{X}_{q}=\left\{Z(X, n): n<\omega, X \in \mathfrak{X}_{n}\right\} \cup \bigcup_{n<\omega} \mathfrak{X}_{n}
$$

is the required extension. Using some enumeration device, to take care of (xi), we need to provide for each $n<\omega$ and $X \in \mathfrak{X}_{n}$ an integer $m>n$ such that the definition of $p_{m+1}, p_{m+2}, \ldots$ will ensure (xi) for this $X$. Let

$$
X_{1}=\left\{a \in X: x_{m} \backslash x_{n} \subseteq A_{a}\right\} .
$$

By the assumption $p_{m} \leq p_{n}$, this set is cofinal in $[\mathcal{I}]^{\omega}$. Since $\mathcal{I}$ is a P-ideal there is $A_{M} \subseteq M \cap \theta$ in $\mathcal{I}$ such that $A \subseteq{ }^{*} A_{M}$ for all $A \in \mathcal{I} \cap M$. Since $X_{1}$ is $\subseteq$-cofinal in $[\mathcal{I}]^{\omega}$ there is a finite set $F_{M} \subseteq M \cap \theta$ such that

$$
X_{2}=\left\{a \in X_{1}: A_{M} \subseteq A_{a} \cup F_{M}\right\}
$$

is cofinal in $[\mathcal{I}]^{\omega}$. So if we are able to find an extension $p_{m+1}$ of $p_{m}$ in $\mathcal{D}_{m} \cap M$ such that $x_{m+1} \backslash x_{m}$ is included in $\left(A_{M} \cap B\right) \backslash F_{M}$ (and also keep this true about any $x_{i+1} \backslash x_{i}$ for $\left.i \geq m\right)$, this will ensure that the set $Z(X, n)$ includes the cofinal set $X_{2}$ so itself will be cofinal in $[\mathcal{I}]^{\omega}$. So it really suffices to show how to satisfy the following two demands for a given set $A \subseteq M \cap \theta$ in $\mathcal{I}$ with the property that $C \subseteq^{*} A$ for all $C \in \mathcal{I} \cap M$ :

$$
\begin{gathered}
p_{m+1} \in \mathcal{D}_{m} \cap M, \\
x_{m+1} \backslash x_{m} \subseteq A .
\end{gathered}
$$

Suppose such an extension $p_{m+1}$ cannot be found. Let $Y_{0}$ be the collection of all $a \in[\mathcal{I}]^{\omega}$ such that for some finite set $F \subseteq A_{a}$ there is no $p \in \mathcal{D}_{m}$ extending $p_{m}$ such that $x_{p} \backslash x_{p_{m}}$ is a subset of $A_{a} \backslash F$. Clearly $Y_{0} \in M$. Note that every $a \in[\mathcal{I}]^{\omega}$ which belongs to $M$ is a member of $Y_{0}$ since we can take $F=A_{a} \backslash A$. It follows that $Y_{0}=[\mathcal{I}]^{\omega}$. By the Pressing Down Lemma, applied in $M$, there is a finite set $F_{0}$ in $M$ and a stationary subset $S$ of $[\mathcal{I}]^{\omega}$ in $M$ such that $F_{0}$ witnesses $a \in Y_{0}$ for all $a \in S$. Note that

$$
q_{1}=\left\langle x_{p_{m}}, \mathfrak{X}_{p_{m}} \cup\{S\}\right\rangle
$$

is a member of $\mathcal{P} \cap M$ which extends $p_{m}$ so by Lemma 1 , applied in $M$, there is a $\beta$ in $M \cap\left(\max \left(x_{p_{m}} \cup F_{0}\right), \theta\right)$ such that

$$
q_{2}=\left\langle x_{p_{m}} \cup\{\beta\}, \mathfrak{X}_{q_{1}} \cup\left\{\left\{a \in X: \beta \in A_{a}\right\}: X \in \mathfrak{X}_{q_{1}}\right\}\right\rangle
$$


belongs to $\mathcal{P} \cap M$ and it extends $q_{1}$. Since $\mathcal{D}_{m}$ is a dense open subset of $\mathcal{P}$ which belongs to $M$ there is $q_{3} \leq q_{2}$ in $\mathcal{D}_{m} \cap M$. Applying the definition of $q_{3} \leq q_{1}$ to $X=S \in \mathfrak{X}_{q_{1}}$ we conclude that

$$
T=\left\{a \in S: x_{q_{3}} \backslash x_{p_{m}} \subseteq A_{a}\right\}
$$

is cofinal in $[\mathcal{I}]^{\omega}$. Choose $a$ in $T$. Since $\beta=\min \left(x_{q_{3}} \backslash x_{p_{m}}\right)$ the set $x_{q_{3}} \backslash x_{p_{m}}$ does not intersect the finite set $F_{0}$ so from $x_{q_{3}} \backslash x_{p_{m}} \subseteq A_{a}$ we conclude $x_{q_{3}} \backslash x_{p_{m}} \subseteq A_{a} \backslash F_{0}$, contradicting the fact that $F_{0}$ witnesses $a \in Y_{0}$. This finishes the proof of Lemma 2 .

Note that we have just shown the following.

Lemma 3. The Proper Forcing Axiom implies (*).

Let us now recall the notion of $\alpha$-proper forcing for a countable ordinal $\alpha$ saying that, given an $\in$-chain of length $\alpha$ of countable elementary sumbodels of some large enough structure $H_{\kappa}$ containing our forcing notion, an arbitrary condition from the smallest model can be extended to a condition that is simultaneously generic over all the models of the chain. It turns out that with very little extra work one can show that our forcing notion $\mathcal{P}$ satisfies this stronger condition of properness. This stronger property of $\mathcal{P}$ will be needed below in Lemma 7 .

Lemma 4. $\mathcal{P}$ is $\alpha$-proper for every countable ordinal $\alpha$.

Proof. This is done by induction on $\alpha$ using the following inductive hypothesis:

(xiv) For every continuous $\in$-chain $M_{\xi}(\xi \leq \alpha)$ of countable elementary submodels of $H_{\kappa}$, every $p_{0} \in \mathcal{P} \cap M_{0}$ and every set $B \subseteq M_{\alpha} \cap \theta$ such that $A \subseteq_{*} B$ for all $A \in \mathcal{I} \cap M_{\alpha}$ there is a $q \in \mathcal{P}$ extending $p_{0}$ which is $M_{\xi}$-generic for all $\xi \leq \alpha$ and which has the property that $x_{q} \backslash x_{p_{0}} \subseteq B$.

We have seen the case $\alpha=0$ during the course of the proof of Lemma 2 . Suppose first that $\alpha$ is a successor ordinal and let $a=\mathcal{I} \cap M_{\alpha-1}$. Note that $A_{a}$ belongs to $M_{\alpha}$ being definable from $<_{w}$ and $a$ which belong to $M_{\alpha}$. Therefore, this step follows from the inductive hypothesis applied to the set $B \cap A_{a}$ (which, being a cofinite subset of $A_{a}$, belongs to $M_{\alpha}$ ) inside the model $M_{\alpha}$ and then applying the case of a single model. Assume now that $\alpha$ is a limit ordinal and fix an increasing sequence $\alpha_{i}(i<\omega)$ cofinal in $\alpha$. For $i<\omega$, let $a_{i}=M_{\alpha_{i}} \cap \mathcal{I}$ and let $B_{i}=B \cap A_{a_{i}}$. Note that $B_{i} \in M_{\alpha_{i}+1}$. Recursively on $i$, starting from $p_{0}$, we build a decreasing sequence of conditions $p_{i+1} \in M_{\alpha_{i}+1} \cap \mathcal{P}(i<\omega)$ such that

$$
x_{p_{i+1}} \backslash x_{p_{i}} \subseteq B_{i} \backslash F_{i},
$$


where $F_{i}$ is a finite subset of $B_{i}$ given to us by some bookkeeping device in order to make sure that any set $X$ appearing in some $\mathfrak{X}_{p_{n}}$ will have its set $Z(X, n)$ (see (xi) above) cofinal in $[\mathcal{I}]^{\omega}$. Note that there is no problem in getting $p_{i+1}$ from $p_{i}$ since working in $M_{\alpha_{i}+1}$ we can apply the inductive hypothesis to $p_{i},\left\langle M_{\xi}: \xi \leq \alpha_{i}\right\rangle$ and $B_{i} \backslash F_{i}$ which all belong to $M_{\alpha_{i}+1}$. Once this is done the pair $q=\left\langle x_{q}, \mathfrak{X}_{q}\right\rangle$ obtained by letting

$$
x_{q}=\bigcup_{n<\omega} x_{p_{n}} \text { and } \mathfrak{X}_{q}=\left\{Z(X, n): n<\omega, X \in \mathfrak{X}_{p_{n}}\right\} \cup \bigcup_{n<\omega} \mathfrak{X}_{p_{n}}
$$

will be a condition of $\mathcal{P}$ which extends all $p_{i}$ 's and satisfies the conclusion of Lemma 3.

Starting again from the basic idea of the proof of Lemma 2, we now establish an even stronger completeness property of our forcing notion $\mathcal{P}$. To state this we need yet another basic notion from $[7, \mathrm{~V}]$ which, for the convenience of the reader, will be defined during the course of the proof of the following lemma.

LEMMA 5. $\mathcal{P}$ is complete with respect to some simple $\sigma$-complete completeness system.

Proof. We need to find a second order formula $\Psi\left(Y_{1}, Y_{2} ; y_{1}, y_{2}, y_{3}\right)$ which with every triple $\left\langle M, \mathcal{P}, p_{0}\right\rangle$, where $M$ is a countable elementary submodel of $H_{\kappa}$, associates the family

$$
\mathcal{G}_{C}^{\Psi}=\left\{G \in \operatorname{Gen}\left(M, \mathcal{P}, p_{0}\right): M \models \Psi\left(G, C, \mathcal{P}, p_{0}, \vec{a}\right)\right\},
$$

for $C$ an arbitrary subset of $M$, that forms a $\sigma$-complete filter base on the set $\operatorname{Gen}\left(M, \mathcal{P}, p_{0}\right)$ of all $(M, \mathcal{P})$-generic subsets of $\mathcal{P} \cap M$ that have extensions in $\mathcal{P}\left(\leq p_{0}\right)$. Here $\vec{a}$ is a fixed parameter from $H_{\kappa}$. To see what $\Psi$ should say consider a triple $\left(M, \mathcal{P}, p_{0}\right)$. Suppose $C$ is a subset of $M$ which codes, in some standard way, the following objects:

(xvi) an enumeration $\left\{\mathcal{D}_{n}\right\}$ of all dense open subsets of $\mathcal{P}$ which belong to $M$,

(xvii) an enumeration $\left\{X_{n}\right\}$ of all cofinal subsets of $\mathcal{I}$ which belong to $M$, (xviii) a function $F: \omega \rightarrow[M \cap \theta]<\omega$,

(xix) a subset $B \subseteq M \cap \theta$ such that $A \subseteq{ }^{*} B$ for all $A \in \mathcal{I} \cap M$.

We let $\Psi\left(G, C ; \mathcal{P}, p_{0}, \mathcal{I}\right)$ say that the decoding of $C$ gives us objects of (xvi)-(xix), that $G$ is a filter generated by a decreasing sequence $\left\{p_{n}\right\}$ of conditions which starts with $p_{0}$ such that for all but finitely many $m, p_{m+1}$ belongs to $\bigcap_{i<m} \mathcal{D}_{i}$ and that $x_{p_{m+1}} \backslash x_{p_{m}}$ is a subset of $B$ disjoint from any finite set $F \subseteq M \cap \theta$ of the following form: there is $i \leq m$ such that $X_{i}$ belongs to some $\mathfrak{X}_{p_{j}}(j \leq m)$ and if $k$ is the minimal such $j$, then $F=F(l)$ 
where $l$ is the minimal integer such that

$$
X_{l}=\left\{a \in X_{i}: x_{p_{m}} \backslash x_{p_{k}} \subseteq A_{a}\right\} .
$$

This definition is based on the proof of Lemma 2 where the function $F$ : $\omega \rightarrow[M \cap \theta]^{<\omega}$ is given by letting $F(i)$ be the minimal finite set $E$ such that

$$
X_{l}=\left\{a \in X_{i}: A_{M} \subseteq A_{a} \cup E\right\}
$$

is cofinal in $[\mathcal{I}]^{\omega}$. Thus any set $C \subseteq M$ whose decoding gives us this particular $F: \omega \rightarrow[M \cap \theta]^{<\omega}$ and whose set $B$ of (xix) is equal to $A_{M}$ will have the property that any $G$ such that

$$
M \mid=\Psi\left(G, C ; \mathcal{P}, p_{0}, \mathcal{I}\right)
$$

will be an $M$-generic filter of $\mathcal{P} \cap M$ containing $p_{0}$ and having extensions in $\mathcal{P}$. In other words, such $X$ will be a witness of $\mathcal{P}$ being complete with respect to the completeness system defined by $\Psi$.

It is not difficult to verify that the system which $\Psi$ defines is in fact $\sigma$ complete since the diagonalization procedure presented above in the proof of Lemma 2 is flexible enough to satisfy an arbitrary sequence $\left\{B_{n}\right\}$ of sets as in (x) rather than a single $B \subseteq M \cap \theta$ with the property $A \subseteq \subseteq^{*} B$ for all $A \in \mathcal{I} \cap M$.

We have already mentioned that the case when the ideal $\mathcal{I}$ lives on $\theta=\omega_{1}$ is in some sense special and that the consistency of $(*)$ for $S=\omega_{1}$ does not require large cardinals. This comes from the fact that in this case the poset $\mathcal{P}=\mathcal{P}_{\mathcal{I}}$ satisfies some strong chain condition that allows it to be iterated preserving cardinals. Unfortunately, the chain condition Lemma 3.6 of [1] has the implicit assumption of $\mathrm{CH}$ as the very definition of the poset $\mathcal{P}_{\mathcal{I}}$ uses this hypothesis. Since there might be some reasons to consider the present version of $\mathcal{P}_{\mathcal{I}}$ in cases when $\mathrm{CH}$ fails, we sketch a proof of the chain condition lemma which does not use any assumptions about the continuum.

Lemma 6. Suppose $\mathcal{I}$ is a P-ideal of countable subsets of $\omega_{1}$ such that $\omega_{1}$ cannot be decomposed into countably many sets orthogonal to $\mathcal{I}$. Then the corresponding poset $\mathcal{P}=\mathcal{P}_{\mathcal{I}}$ satisfies the properness isomorphism condition.

P r o of. This means that if we are given two countable elementary submodels $M_{0}$ and $M_{1}$ of some large enough structure $H_{\kappa}$ such that $\mathcal{P} \in$ $M_{0} \cap M_{1}$ and an isomorphism $h$ between $M_{0}$ and $M_{1}$ which is equal to the identity on $M_{0} \cap M_{1}$, then for every $p \in \mathcal{P} \cap M_{0}$ there is a condition $q \in \mathcal{P}$ which extends both $p$ and $h(p)$ such that $q$ is $M_{0}$-generic and such that $q$ forces that the mapping $h$ sends $\dot{G} \cap M_{0}$ isomorphically onto $\dot{G} \cap M_{1}$, where $\dot{G}$ is the canonical name for the generic filter of $\mathcal{P}$.

The condition $q$ is obtained as an extension of a sequence $p_{n}(n<\omega)$ of elements of $\mathcal{P} \cap M_{0}$ which starts with $p_{0}=p$ and which is obtained via a diagonalization procedure similar to that in the proof of Lemma 2 . The only 
new feature is that now when moving from $p_{m}$ to $p_{m+1}$ we have to take care not only of cofinal sets appearing in certain $\mathfrak{X}_{p_{n}}$ 's but also of their $h$-images. Thus, at a given stage $m$ where we take care of some $X \in \mathfrak{X}_{p_{i}}(i \leq m)$, we additionally take care of $h(X)$. Note that $h\left(p_{m}\right)$ extends $h\left(p_{i}\right)$ and that since $h$ is the identity on $\mathcal{I}$ (and this is the point where the assumption that $\mathcal{I}$ consists of countable subsets of $\omega_{1}$ is used), we have

$$
x_{h\left(p_{m}\right)}=x_{p_{m}} \text { and } x_{h\left(p_{i}\right)}=x_{p_{i}} .
$$

It follows that the set

$$
X_{1}=\left\{a \in h(X): x_{p_{m}} \backslash x_{p_{i}} \subseteq A_{a}\right\}
$$

is cofinal in $[\mathcal{I}]^{\omega}$. So there is a finite set $F \subseteq \delta=M_{0} \cap \omega_{1}=M_{1} \cap \omega_{1}$ such that

$$
X_{2}=\left\{a \in X_{1}: A_{\left(M_{0} \cap \mathcal{I}\right)} \subseteq A_{a} \cup F\right\}
$$

is cofinal in $[\mathcal{I}]^{\omega}$. This will be an additional finite set which we will have to avoid while constructing $p_{n}(n>m)$. When this is done we form a condition $q=\left(x_{q}, \mathfrak{X}_{q}\right)$ of $\mathcal{P}$ by letting $x_{q}$ be the union of the $x_{p_{n}}$ 's and

$$
\begin{aligned}
\mathfrak{X}_{q} & =\bigcup_{n<\omega}\left(\mathfrak{Y}_{n} \cup \mathfrak{Z}_{n}\right), \quad \text { where } \\
\mathfrak{Y}_{n} & =\mathfrak{X}_{p_{n}} \cup\left\{\left\{a \in X: x_{q} \backslash x_{p_{n}} \subseteq A_{a}\right\}: X \in \mathfrak{X}_{p_{n}}\right\}, \\
\mathfrak{Z}_{n} & =h^{\prime \prime} \mathfrak{X}_{p_{n}} \cup\left\{\left\{a \in h(X): x_{q} \backslash x_{p_{n}} \subseteq A_{a}\right\}: X \in \mathfrak{X}_{p_{n}}\right\} .
\end{aligned}
$$

Then $q$ is an $M_{0}$-generic condition which extends not only the $p_{n}$ 's but also their $h$-images $h\left(p_{n}\right)^{\text {'s, }}$, and therefore forces that $\dot{G} \cap M_{0}$ is generated by the sequence $\left\{p_{n}\right\}$ while $\dot{G} \cap M_{1}$ is generated by the sequence $\left\{h\left(p_{n}\right)\right\}$. This finishes the proof.

We are now in a position to complete the consistency proof of $(*)$ with GCH assuming the consistency of the existence of a supercompact cardinal.

LEMMA 7. If there is a supercompact cardinal then there is a proper forcing notion which simultaneously forces (*) and the GCH.

Proof. As indicated in the introduction to this section, the general outline of the proof is a well familiar one. We take a supercompact cardinal $\kappa$ and if necessary go to a forcing extension so that we moreover have the GCH. We choose a Laver sequence $f: \kappa \rightarrow V_{\kappa}$ with the property that for every set $x$ and every large enough cardinal $\lambda$ there is an elementary embedding $j: V \rightarrow M$ with critical point $\kappa$ such that $[M]^{\lambda} \subseteq M, j(\kappa)>\lambda$ and $(j f)(\kappa)=x$. Using $f$ we build a countable support iteration

$$
\left\langle\mathcal{P}_{\xi}, \dot{\mathcal{Q}}_{\xi}: \xi \leq \kappa\right\rangle
$$

of posets that belong to $V_{\kappa}$ such that with probability one each $\dot{\mathcal{Q}}$ is $\alpha$-proper for all $\alpha<\omega_{1}$ and complete relative to some simple $\sigma$-complete complete- 
ness system so that we can apply the basic result of $[7, \mathrm{~V}]$ and have the conclusion that no new reals are added at any stage. Since, clearly, $\mathcal{P}_{\kappa}$ satisfies the $\kappa$-chain condition the forcing extension preserves all cardinals $\geq \kappa$ and has its second uncountable cardinal equal to $\kappa$. To make sure $\mathcal{P}_{\kappa}$ forces (*), we choose $\dot{\mathcal{Q}}_{\xi}$ as follows. Suppose that $f(\xi)$ is equal to $(\dot{\mathcal{I}}, \dot{\theta})$ where

(xxi) $\mathcal{I}$ is a $\mathcal{P}_{\xi}$-name for a $\mathrm{P}$-ideal of countable subsets of the ordinal $\dot{\theta}$ such that $\dot{\theta}$ cannot be decomposed into countably many subsets orthogonal to $\dot{\mathcal{I}}$ but every smaller ordinal can.

In this case we let $\dot{\mathcal{Q}}_{\xi}$ be the $\mathcal{P}_{\xi}$-name for the poset $\mathcal{P}_{\dot{\mathcal{I}}}$ described above, which by Lemma 1 introduces an uncountable subset $A$ of $\dot{\theta}$ such that $[A]^{\omega} \subseteq$ $\dot{\mathcal{I}}$. If $f(\xi)$ is not a pair of names of this form we let $\dot{\mathcal{Q}}_{\xi}$ be the $\mathcal{P}_{\xi}$-name for the trivial poset. By Lemmas 3 and 4 we see that with probability one $\dot{\mathcal{Q}}_{\xi}$ is $\alpha$-proper for all $\alpha<\omega_{1}$ and is complete relative to some simple $\sigma$-complete completeness system, so we are preserving the inductive hypothesis with this choice of the name.

To see that $\mathcal{P}_{\kappa}$ forces $(*)$, consider a $\mathcal{P}_{\kappa}$-name $\dot{\mathcal{I}}$ for a P-ideal of countable subsets of some ordinal $\dot{\theta}$ with the property that $\dot{\theta}$ cannot be decomposed into countably many sets orthogonal to $\dot{\mathcal{I}}$ but every smaller ordinal can. By the property of the Laver sequence $f$, we find a large enough regular cardinal $\lambda$ and an elementary embedding $j: V \rightarrow M$ such that $\kappa$ is the critical point of $j,[M]^{\lambda} \subseteq M, j(\kappa)>\lambda$ and $(j f)(\kappa)=(\dot{\mathcal{I}}, \dot{\theta})$. In the model $M$ we find that $j\left(\left\langle\mathcal{P}_{\xi}, \dot{\mathcal{Q}}_{\xi}: \xi<\kappa\right\rangle\right)=\left\langle\mathcal{P}_{\xi}, \dot{\mathcal{Q}}_{\xi}: \xi<j(\kappa)\right\rangle$ is a countable support iteration whose initial part up to $\kappa$ agrees with our initial iteration. Applying the same rule in $M$ to choosing $\dot{\mathcal{Q}}_{\xi}$ at stage $\xi=\kappa$ we enter into its nontrivial case $(j f)(\kappa)=\langle\dot{I}, \dot{\theta}\rangle$. So $\dot{\mathcal{Q}}_{\kappa}$ is a $\mathcal{P}_{\kappa}$-name for the poset $\mathcal{P}_{\dot{\mathcal{I}}}$. By Lemma 1 , forcing with $\dot{\mathcal{Q}}_{\kappa}$ introduces an uncountable subset $\dot{A}$ of $\theta$ such that $[A]^{\omega} \subseteq \dot{\mathcal{I}}$. Using the $\kappa$-chain condition of $\mathcal{P}_{\kappa}$ one sees that $j$ naturally extends to an elementary embedding $j: V^{\mathcal{P}_{\kappa}} \rightarrow M^{\mathcal{P}_{j(\kappa)}}$. Recall that with probability one $\lambda$ is much larger than $\dot{\theta}$ and that $M$ is closed under $\lambda$-sequences. So in particular the mapping $i=j\lceil\lambda$ is a member of $M$. By the elementarity of the embedding $j: V^{\mathcal{P}_{\kappa}} \rightarrow M^{\mathcal{P}_{j(\kappa)}}$ (and the fact that no new countable sets of ordinals have ever been added) we see that the mapping $i$ moves the set $\dot{A}$ (which $M^{\mathcal{P}_{j(\kappa)}}$ knows already at stage $\kappa+1$ ) to an uncountable set $\dot{B}$ with the property that $[\dot{B}]^{\omega} \subseteq j(\dot{\mathcal{I}})$. So $M^{\mathcal{P}_{j(\kappa)}}$ sees the first alternative of $(*)$ for $j(\mathcal{I})$ satisfied, and so by the elementarity of the embedding $j$, the extension $V^{\mathcal{P}_{\kappa}}$ must see the first alternative of $(*)$ holding for $\dot{\mathcal{I}}$. This completes the proof.

\section{References}

[1] U. Abraham and S. Todorčević, Partition properties of $\omega_{1}$ compatible with $C H$, Fund. Math. 152 (1997), 165-181. 
[2] K. J. Devlin, The Yorkshireman's guide to proper forcing, in: Surveys in Set Theory, A. R. D. Mathias (ed.), Cambridge Univ. Press, 1983, 60-105.

[3] F. Hausdorff, Summen von $\aleph_{1}$ Mengen, Fund. Math. 26 (1936), 241-255.

[4] J. Hirschorn, Random trees under CH, preprint, 1999.

[5] R. B. Jensen, The fine structure of the constructible hierarchy, Ann. Math. Logic 4 (1972), 229-308.

[6] R. Laver, Making supercompactness indestructible under $\kappa$-directed forcing, Israel J. Math. 29 (1978), 385-388.

[7] S. Shelah, Proper Forcing, Springer, 1982.

[8] S. Todorčević, Trees and linearly ordered sets, in: Handbook of Set-Theoretic Topology, K. Kunen and J. E. Vaughan (eds.), North-Holland, 1984, 235-293.

[9] -, Partitioning pairs of countable ordinals, Acta Math. 159 (1987), 261-294.

[10] -, Partition Problems in Topology, Amer. Math. Soc., Providence, 1989.

[11] - Some applications of $S$ and L combinatorics, Ann. New York Acad. Sci. 705 (1993), 130-167.

Université Paris 7, C.N.R.S.

UPRESA 7056

2, Place Jussieu

72251 Paris Cedex 05, France

E-mail: stevo@math.jussieu.fr stevo@math.toronto.edu 